Washington for the use of a malt amylase preparation which had been purified in connection with investigations conducted by aid of its grants. NEW YORK CITY.

[CONTRIBUTION FROM THE LABORATORY OF THE NORThWESTERN UNIVERSITY MEDICAL, ScHOOL.]

\title{
ON THE ASSUMED DESTRUCTION OF TRYPSIN BY PEPSIN AND ACID. III. OBSERVATIONS ON MEN. ${ }^{1}$ \\ By J. H. LoNg and Mary Hull. \\ Received May 26, 1917.
}

In two earlier publications we have given data on this problem obtained from experiments in vitro and from observations on dogs. ${ }^{2}$ From both lines of investigation it appeared that trypsin is much more resistant to the action of pepsin under certain conditions than was formerly supposed. We are now attempting to duplicate some of the experiments carried out on the dogs through somewhat similar experiments carried out on the human subject.

The plan observed is essentially this. Following a test meal the whole stomach contents, as far as possible, are drawn off after a proper interval and examined for acid, bile, pepsin and possible tryptic action. If the stomach is found to behave normally then, in the observation proper, some powdered trypsin is given along with the test meal and after a time the contents are drawn off as before and examined for the persistence of the tryptic action. In the preliminary experiments where no trypsin is ingested the tryptic proteolysis found is very slight, usually, and may depend on a behavior normal to the stomach, or it may come from the regurgitation of intestinal contents holding small amounts of the ferment. The presence of bile would suggest the possibility of this. The proof of the presence of trypsin in the stomach contents is found in the ability of the collected liquid to digest fibrin under conditions where pepsin present can not act, that is, in a medium of a slight degree of alkalinity. The extent of digestion through a period of three hours is measured, finally, by the formaldehyde amino acid titration.

As subjects for our observations we have used some cases from the hospitals connected with the School and some students who were willing to submit to the tests through periods long enough to have value. Several series of tests were begun and abandoned because the subjects found it irksome to take the Rehfuss tube daily. The results of the observations satisfactorily completed are given below in tabular form. While most

1 This investigation has been made with the assistance of a grant from the Committee on Therapeutic Research, Council on Pharmacy and Chemistry, American Medical Association.

2 Thrs JOURNAL, 38, I620 (I916); 39, I62 (I917). 
of the subjects were hospital cases they were fully suitable for such investigations because no alimentary derangement was involved and the appetite and food digestion were always satisfactory.

The following general explanations will aid in understanding the tables. In each case the number of the subject, date and character of test meal and ingestion of trypsin (when given) are stated first. After the meal an interval of half an hour or more was allowed to elapse before the tube was inserted to bring up contents left. In this time the trypsin would be exposed to the action of pepsin and acid, but always in the presence of more or less protein. The length of this interval is given and in the next column the volume of contents secured by the Rehfuss tube. As everything in the test meal is in a very finely divided condition practically the whole of the contents may be drawn up, and this was usually the case. With small portion of the contents tests for free and total $\mathrm{HCl}$ were made; the results are quantitative, but not of a high degree of accuracy, as this was not necessary for our purpose. They were secured by the usual double titration with methyl orange and phenolphthalein and are given in the next columns. The presence or absence of bile is next indicated.

In order to determine the presence of trypsin in the stomach contents a measured volume was incubated with a gram of prepared dried fibrin in a medium of known, slightly alkaline, reaction through a three-hour period at $40^{\circ}$. The volume of contents taken for the test was usually $25 \mathrm{cc}$. and it was first made neutral to litmus before mixing with the $20 \mathrm{cc}$. of 0.067 molar phosphate solution as the alkaline substrate. This solution has a reaction given by $\mathrm{P}_{\mathrm{H}}=7.7$. The mixture is diluted to 5o cc. before incubation. In a parallel or blank determination the same conditions are observed with this exception, that the ferment in the contents is destroyed by heat before mixing. After the incubation both liquids are treated with baryta solution to remove phosphates, made up to $100 \mathrm{cc}$. and filtered. A $50 \mathrm{cc}$. portion, in each case, is neutralized to litmus with $\mathrm{HCl}$, treated with formaldehyde and titrated in the usual way with $0.2 N$ sodium hydroxide. The blank titration measures the preformed amino acid present. This subtracted from the other shows the acid liberated in the actual digestion. This difference is stated in a separate column and if it is multiplied by 2 we have the measure of the digestion for the whole roo ce. of the diluted incubated mixture, or for the $25 \mathrm{cc}$. or other volume of contents taken. From this the value for the total contents withdrawn is calculated and this is stated in the table. Finally, a positive test for pepsin was always found. This was always made by aid of small albumin tubes incubated with a little of the liquid, but it is not detailed in the table.

The table shows a wide range of digestive values. In not a few cases the alkaline digestion, with no added trypsin, is quite appreciable. This 
usually occurs in the portions of the contents in which the presence of bile is indicated, and it is probable that here some pancreatic trypsin has come into the stomach at the same time. It will be further noted that the highest results for amino acid liberation occur in a number of cases where bile is indicated and trypsin ingested at the same time. In all these the content of hydrochloric acid in the free state is appreciable, while the combined acid is higher.

But there are some instances where there is no evidence of bile in which, without added trypsin, we find a fairly good tryptic digestion later. This shows, for example, in Case $\mathrm{H}$ in the first days of the tests. The subject was admitted to the hospital on account of lameness due apparently to rheumatism.' No lack of gastric activity was suspected until after the beginning of these observations. The large volume of liquid which could be secured indicated that the stomach was emptied but slowly, and as no free acid could be found by the methyl orange titration while the combined acid was quite negligible it became evident that no peptic digestion would be possible. Appropriate tests disclosed the presence of pepsin each day. There appeared to be some tryptic acitivity here as shown in the tests of April 5th and 6th. The source of this activity is problematical, but there is this possibility that the stomach itself must secrete trypsin under the conditions which obtain.

When later in this man's case trypsin was administered the motility of the stomach seemed to be greatly increased, with the result that the contents were much more quickly passed out. The volume of liquid which could be secured for the tests was greatly diminished; probably much of the administered trypsin passed along with the chyme. However, as there was no free acid in this case the experience with the subject has no marked bearing on the question of the behavior of trypsin in a more nearly normal stomach.

The results secured from Subject $A$ are very instructive. The free acid is usually low but the data given for the total acid show that there is a good secretion. There is low tryptic activity on July $14^{\text {th }}, 5^{\text {th }}$, 17 th and I8th, but on these dates the acid present is considerable, and this may explain why the effect following the ingestion of trypsin is so slight. On two of the days the combined acid is high, indicating a marked secretion. The conditions for persistence of the trypsin are not, therefore, favorable. But on July Igth and 2 Ist, with the free acid quite low, there is good evidence of the presence of residual trypsin in the stomach, notwithstanding the high content of combined acid. The result for the roth is very marked.

In Subject B the stomach appeared to be so rapidly emptied that the ingested trypsin probably went largely with the chyme. The presence of 


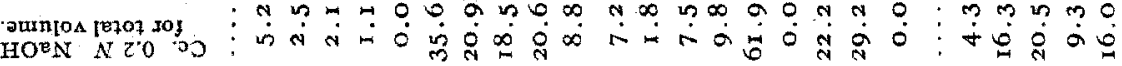

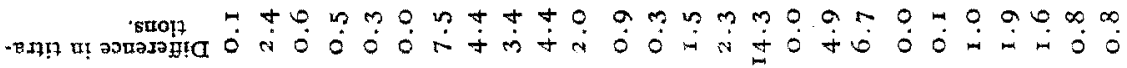

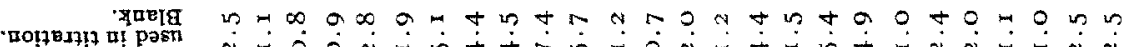

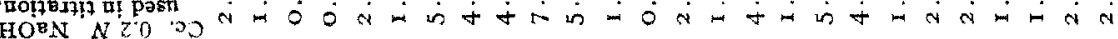

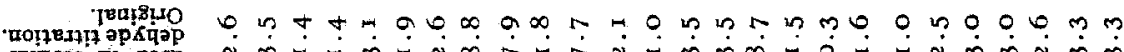

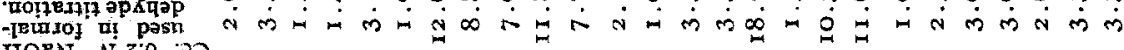
FOEN N 2000

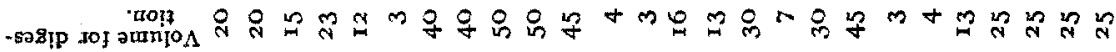

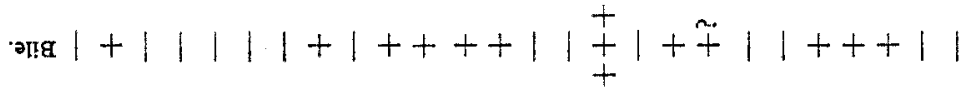

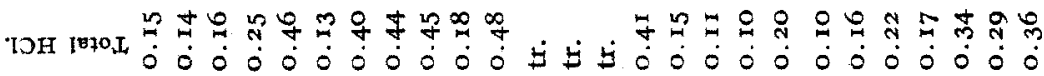

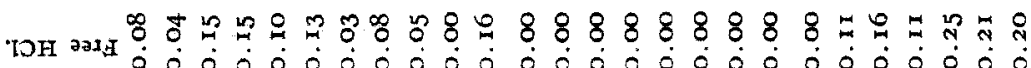
ๆ

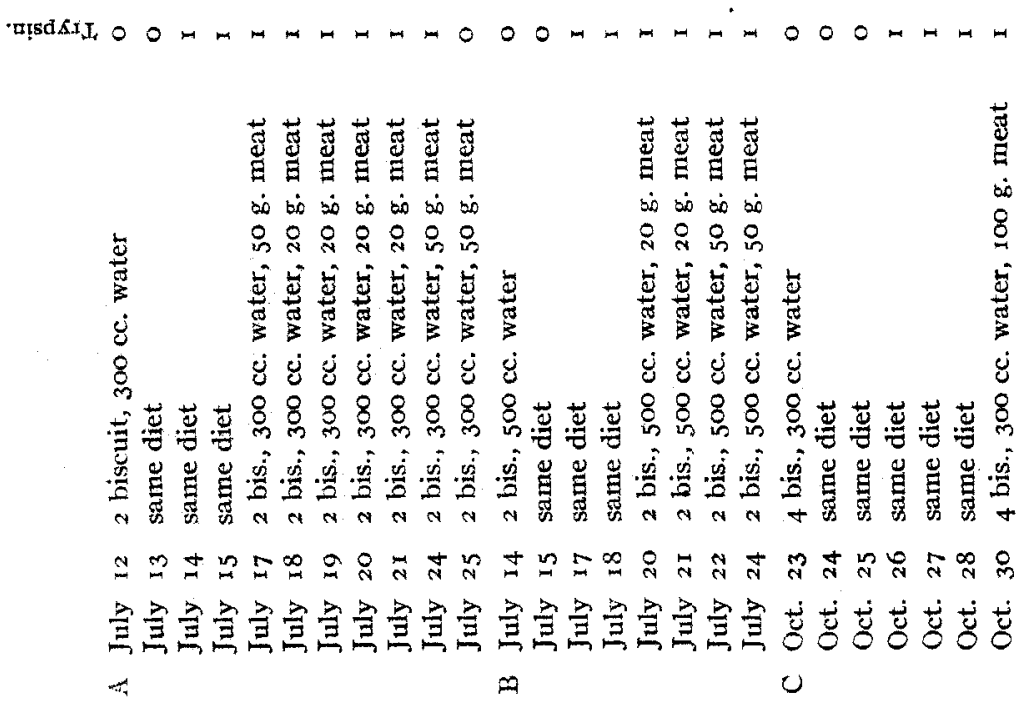


$0+\infty$ ก

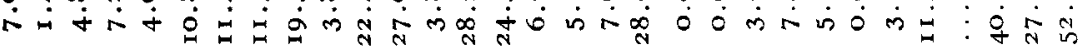

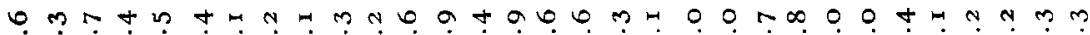

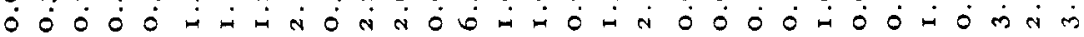

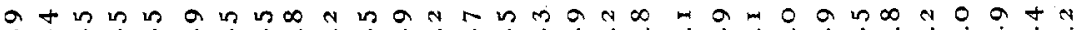
-

in $N$ a 0 mo

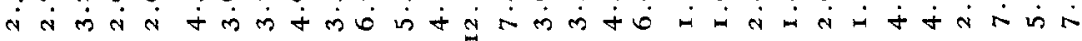

우의 $+++1|+++1+| 1|+| 1|+| 1|+| 1|++1+1|$

순

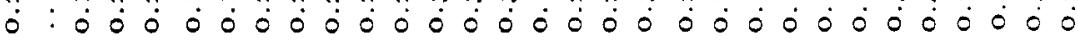

m:

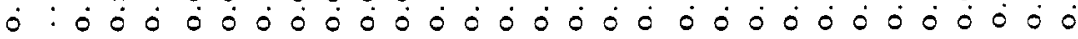

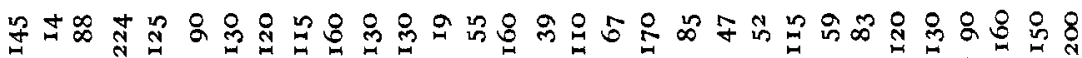

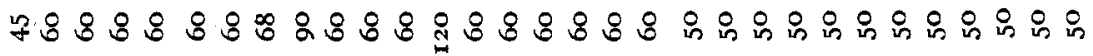

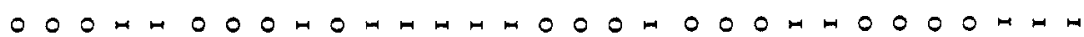

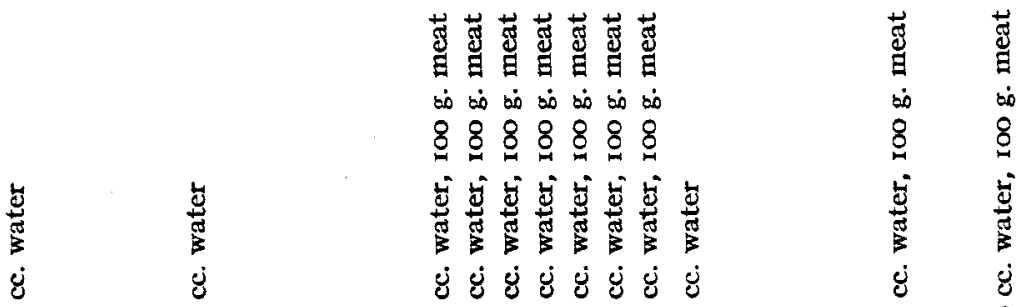

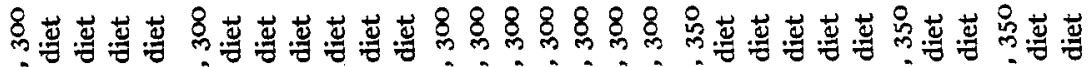

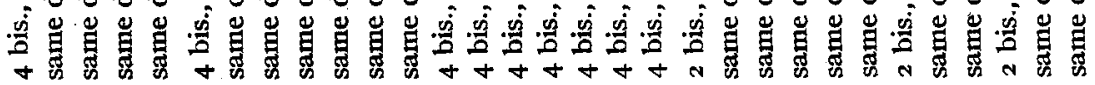
ผ

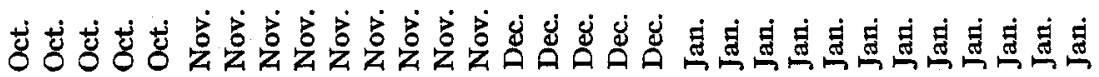
A 


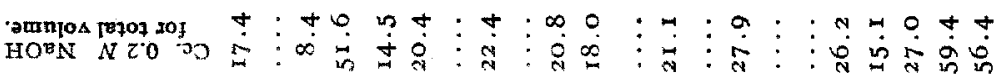

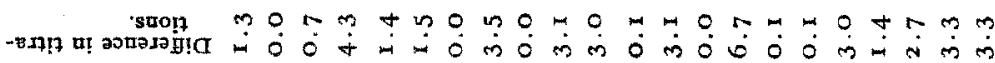

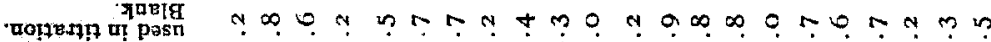

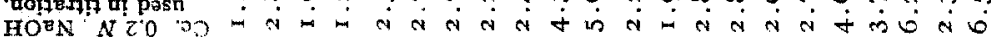

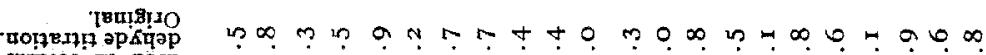

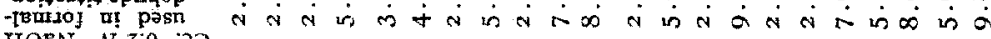
HODN $N Z 0$ OO

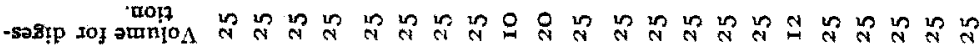

$$
\begin{aligned}
& + \\
& +1++1 \\
& +
\end{aligned}
$$

TDH IR7OI. เон

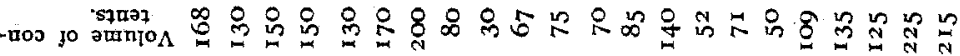

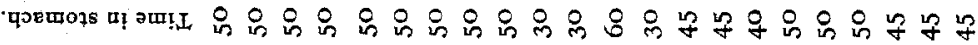

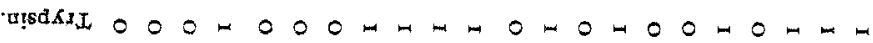

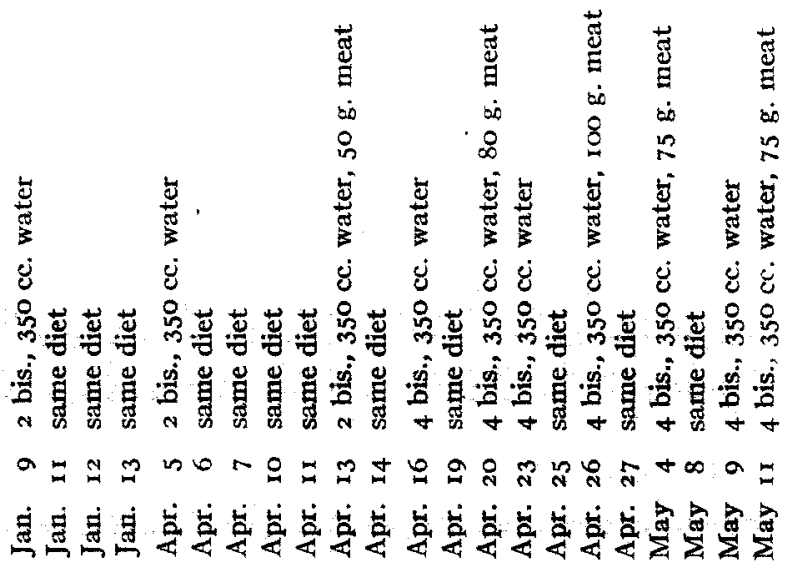
0 
much bile introduced a complication on several of the dates. The very high tryptic activity of July 2 oth is doubtless largely due to the ferment of duodenal regurgitation, as suggested by the marked bile content of the stomach. It will be noticed that there is no free acid in the stomach of this man; the high combined acid of July I8th is not to be explained from data at hand. It is safe to say that we have, however, on this date some indication of the presence of residual trypsin in the small volume of contents secured.

In Subjects $\mathrm{C}$ and $\mathrm{D}$ there is no marked activity on days when there is no bile reaction, but in Subject $\mathrm{E}$ where the free acidity is moderately low we find a strong tryptic action on a number of days when the effect cannot be referred to presence of bile. This is notably true on Nov. 23rd, $25^{\text {th }}$, and $27^{\text {th }}$ and on Dec. Ist and 6th.

In the case of Subject $F$ the tryptic action is highest at times corresponding to the administration of the ferment powder. When bile is not present in the stomach of this man, and no trypsin administered, the final tests give practically negative results. On two dates with a high bile content we note a marked tryptic activity also.

From $\mathrm{G}$ no definite conclusions can be drawn because of the persistent regurgitation of bile.

Subject I furnishes the most tangible data. This is the case of a medical student who for years had suffered from a rather marked hyperacidity. When trypsin is not ingested here the recorded digestion effect is generally low, save on May 4th when bile is recorded. The ingestion of the ferment is invariably accompanied by presence of the same in the gastric contents, and this is true even when the free hydrochloric acid is rather high. With this man we found it desirable to give a fairly strong meat ration to bind a good part of the hydrochloric acid.

Considering the table as a whole it is evident that we have essentially the same kind of proof for the persistence of trypsin in the stomach that we had in the animal experiments. The enzyme is active in both cases after exposure to rather marked acid conditions through a period of half an hour to an hour. In some of the trials we failed to find evidences of the presence of trypsin when we expected to find it, which situation may be illustrated by the tests of July I4th and following days on Subject $A$, to which attention has been called. In addition to a high acidity on these dates time enough had elapsed between ingestion and withdrawal of stomach contents to permit most of the latter to pass into the duodenum, carrying along most of the remaining trypsin, possibly.

Even when a good indication of the presence of bile is in evidence we do not always find the trypsin of regurgitation. There may be several reasons for this. In the first place the duodenal liquid itself may have 
been deficient in the enzyme, and this is a likely supposition. Or, the acidity of the stomach may have been so great as to neutralize the regurgitated liquid and leave in excess enough acid to injuriously affect the intestinal trypsin as it often does the ingested ferment. This seems to be illustrated by Case $\mathrm{C}$, October $25^{\mathrm{th}}$. The failures to recognize duodenal trypsin are apparently as frequent as are those to recognize the ingested trypsin and there are usually good reasons for these failures.

It must be kept in mind that the hydrochloric acid of the stomach is not the only factor in weakening or destroying trypsin. Our previous work is in agreement with the observations of others in showing that the acid alone is without much effect unless the concentration is higher than we are here dealing with. But the addition of pepsin makes an entirely different situation, and the one we are practically considering. In every one of the above cases pepsin was present, providing, consequently, an unfavorable condition for the existence of the trypsin. Yet, we found it in so many instances that we are forced to believe, as the former studies suggested, that its stability in the stomach must be greater than was commonly assumed. Indeed, the high blanks we noted in so many of our observations suggest rather strongly that some tryptic digestion may be possible in the lower acid conditions of the stomach itself. This would follow, in fact, from the results of our investigations on the optinum conditions of acidity or alkalinity for tryptic action. ${ }^{1}$ Such conditions are not far removed from those which sometimes obtain in the stomach, and there may be normally some tryptic digestion in that organ.

\section{Summary.}

The observations of this paper on the human subject justify the conclusions reached in previous work performed in vitro and with dogs that under certain, not unusual, conditions trypsin may resist the action of pepsin and acid in the animal stomach through a considerable period following the ingestion of the ferment.

It is evident that the action of this ingested trypsin is fully as pronounced, in the quantities. used, as is that which seems to be carried back to the stomach, along with bile, by the regurgitation of duodenal fluid but it cannot be said that either effect is very important physiologically. The ingestion of a few milligrams of commercial pancreatin or trypsin could not have great importance as a therapeutic measure.

Some tryptic digestion may undoubtedly take place in a slightly acid medium, and therefore, at times, in the stomach.

Carcago, ILs.

Thrs Journal, 39, 105 I (1917). 